\title{
Arterial stiffness in insulin resistance: The role of nitric oxide and angiotensin II receptors
}

\author{
Divina G Brillante' \\ Anthony J O'Sullivan' \\ Laurence G Howes ${ }^{2}$ \\ 'St. George Clinical School, University \\ of New South Wales, Kogarah, \\ NSW, Australia; ${ }^{2}$ Department \\ of Pharmacology and Therapeutics \\ and Department of Cardiology, \\ Griffith and Bond University, Gold \\ Coast Hospital, Southport, QLD, \\ Australia
}

\begin{abstract}
The insulin resistance syndrome (INSR) is associated with increased cardiovascular risk, and affects up to $25 \%$ of the Australian population aged $>20$ years. Increased arterial stiffness has been proposed as a common pathway by which INSR leads to increased cardiovascular risk. We have reviewed the role of nitric oxide (NO) and angiotensin II receptors in the modulation of arterial stiffness in the setting of insulin resistance. There is emerging evidence that early stages of INSR may be characterized by increased basal nitric oxide activity and increased activity of non-NO vasodilators such as endothelial derived hyperpolarization factor (EDHF) which is manifest by reduced arterial stiffness. Depletion of NO or ineffectiveness of NO mediated vasodilator mechanisms associated with the progression of INSR to type 2 diabetes may result in increased arterial stiffness, which predicts the development of cardiovascular disease. Thus in the early stages of INSR, increased NO and EDHF activity may represent compensatory mechanisms to early vascular damage. The renin-angiotensin system is activated in diseased vascular beds, with up regulation of the two known angiotensin II receptors: the angiotensin II type 1 receptor (AT1R) and the angiotensin II type 2 receptor (AT2R). Increased AT1R mediated activity in the vasculature is central to the development of increased arterial stiffness and is enhanced in INSR states. AT2R activity is increased in early in INSR and may contribute to the apparent increase in basal NO activity. AT1R blockade may therefore be valuable treatment for early INSR as antagonism of AT1 receptors would allow angiotensin II to act unopposed at AT2 receptors.
\end{abstract}

Keywords: arterial stiffness, insulin resistance, nitric oxide, AT1 receptor, AT2 receptor, diabetes, insulin resistance, obesity

\section{Introduction}

Insulin resistance (INSR) is a metabolic abnormality resulting from an impaired physiological response to insulin, and is present in disease states such as type II diabetes mellitus (DM), hypertension, chronic kidney disease and obesity. Of itself, or as part of the "deadly quartet" of cardiometabolic abnormalities making up the INSR syndrome (including hypertension, atherogenic dyslipidemia, and central obesity), INSR is associated with increased cardiovascular risk. Precise figures for the prevalence of INSR is not available, but the INSR syndrome affects up to $25 \%$ of the Australian population aged $>20$ years. ${ }^{1}$ With the median age and weight of the Australian population rising, INSR and its complications are emerging to be a major public health problem.

Increased arterial stiffness has been proposed as a common pathway by which INSR leads to increased cardiovascular risk. ${ }^{2-4}$ It is an established predictor of cardiovascular disease, ${ }^{2-4}$ and is implicated in the development of isolated systolic hypertension: a major cause of cardiovascular morbidity especially in the elderly. Prospective studies have shown that patients with the INSR syndrome have a greater increase in arterial stiffness with age compared with those without. ${ }^{5,6}$ Arterial stiffness is a complex phenomenon, involving structural (extracellular matrix and luminal pressure), cellular 
(endothelial cells and vascular smooth muscle cells) and functional elements of the vessel wall. Structural changes occur in normal individuals from age twenty, ${ }^{7,8}$ with a reduction in elastic tissue, accompanied by its fragmentation and degeneration.

The cellular and functional elements contributing to arterial stiffness is less well established. The endothelium and vascular smooth muscle cells (VSMCs) interact in a complex manner to modulate arterial tone, and the pathways mediating these interactions are still controversial. This review focuses on new concepts involving the role of nitric oxide (NO) and the angiotensin II receptors in the modulation of arterial stiffness, in the early stages of INSR.

NO is synthesized by three isozymes of the enzyme NO synthase (NOS) iNOS, nNOS and eNOS. iNOS is an inducible form of the enzyme that is activated in inflammatory response. nNOS is a constitutive enzyme located in neuronal tissue where NO acts as a modulating neurotransmitter. NO is synthesized in the endothelium by a constitutive NO synthase (e-NOS). ${ }^{9}$ Endothelial production of NO has a vasodilatory effect and anti-atherogenic properties, including inhibition of VSMC proliferation and migration, platelet activation and adhesion and leukocyte adhesion and migration. ${ }^{10}$

Endothelial function can be assessed with a variety of techniques, with forearm blood flow studies after intrabrachial arterial infusion of vasoactive substances being widely accepted as the gold standard. Endothelial dysfunction, demonstrated by reduced vasodilatory response to acetylcholine, have been observed in patients with impaired fasting glycemia ${ }^{11}$ and patients with established type 2 diabetes. ${ }^{12,13}$ In recent years, studies of arterial stiffness changes following systemic administration of vasoactive medications known to affect endothelial function, such as beta- ${ }_{2}$ adrenoceptor agonists and the NO synthase inhibitor L-nitro monomethyl arginine (L-NMMA) have been proposed to be acceptable non-invasive techniques for the measurement of endothelial function. ${ }^{14,15}$ Chowienczyk and colleagues demonstrated significant reductions in reflection index (RI), a measure of small to medium sized arterial stiffness using digital photoplethysmography, after systemic administration of albuterol (which causes vasodilation via beta- ${ }_{2}$ receptor activation of endothelial NO formation) and glyceryl trinitrate (GTN) a direct dilator of VSMCs. Concurrent intravenous administration of the NO-synthase inhibitor, L-NMMA, blunted the RI response to albuterol but not to GTN, ${ }^{14}$ illustrating that small to medium-sized arterial stiffness is at least in part, dependent on the integrity of the endothelial L-arginine-NO pathway (Figure 1). ${ }^{16,17}$

There are several reports of arterial stiffness changes in INSR states ${ }^{18-22}$ and it is generally accepted that increased arterial stiffness heralds the onset of cardiovascular disease In some individuals, this can occur as early as adolescence. ${ }^{23-25}$ This is well documented and will not be explored here further. There is emerging evidence however, of a stage of vascular change in early INSR that is characterized by increased basal NO levels and low to normal arterial stiffness. Mangoni and colleagues observed that young, obese, normotensive, and insulin-resistant subjects had significantly increased radial artery diameter at baseline compared with lean controls ${ }^{26}$ and induction of forearm ischemia by suprasystolic cuff

\section{Small to intermediate-sized artery}

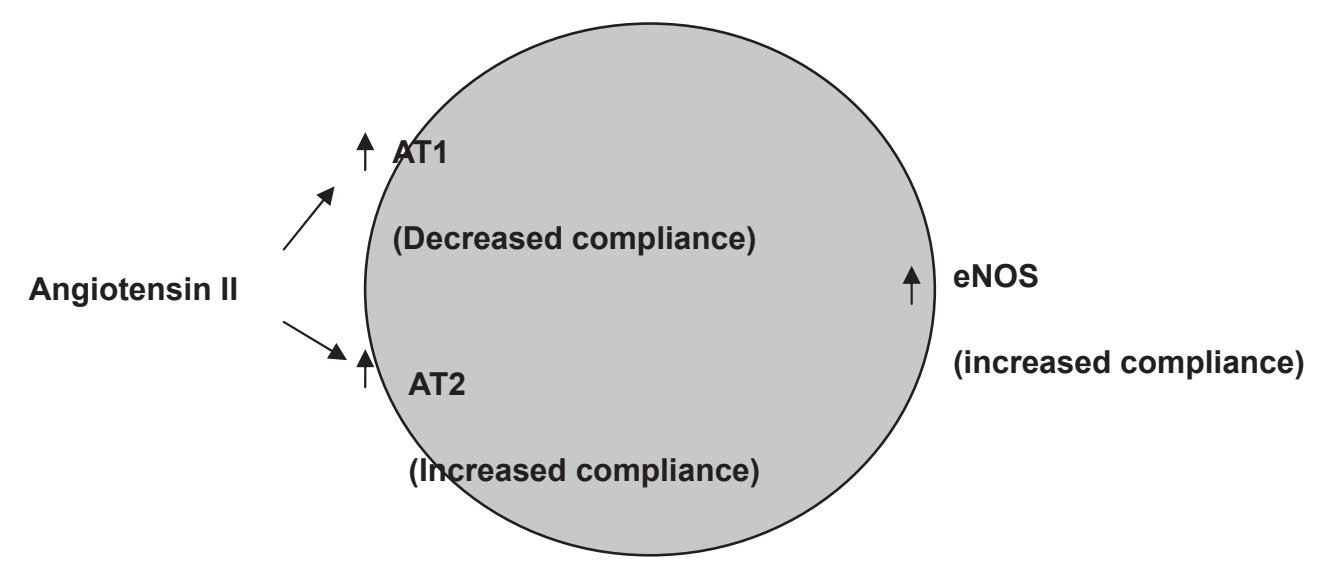

Figure I Changes in responsiveness in small to medium sized artery compliance in patients with early insulin resistance syndrome. There is increased responsiveness to angiotensin II (AT2) mediated by both ATI and AT2 receptors producing decreased and increased compliance respectively. There is also increased basal nitric oxide activity producing an increase in compliance when measured as the reduction in compliance to nitric oxide synthase inhibition. 
compression of the brachial artery produced significantly increased radial artery compliance in these subjects. A similar phenomenon has been described in subjects with mild essential hypertension. ${ }^{27}$ Recently, we observed that normotensive patients with the early INSR syndrome (using the International Diabetes Federation criteria) had significantly reduced baseline arterial stiffness indices (large arterial stiffness index: SI, and reflection index: RI, a measure of small to medium-sized arterial stiffness) using digital photo plethysmography compared with age and sex-matched normal controls (NCs). ${ }^{28}$ Intravenous infusion of L-NMMA $(3 \mathrm{mg} / \mathrm{kg}$ ) in both groups produced a significantly greater increase in small to medium artery stiffness (significantly increased \% change in RI) in INSR subjects compared with the NCs. We concluded that increased basal NO production may contribute to the reduced baseline arterial stiffness indices observed in INSR subjects, and may be a compensatory mechanism to early vascular change in these individuals. To our knowledge, no other studies using other methods of measuring arterial compliance have been performed in patients with INSR.

Similarly, increased basal NO levels have been observed in subjects with early type I and II diabetes mellitus, ${ }^{29-31}$ and may be accompanied by an increase in the activity non-NO vasodilator agents such as endothelial hyperpolarizing factor (EDHF).$^{30}$ Furthermore, increased EDHF activity may play a relatively greater role when NO mechanisms fail ${ }^{32}$ during the progression of early INSR to type 2 diabetes. There is evidence to suggest that the development of microvascular disease in diabetes is accompanied by depletion of basal NO levels and impairment of non-NO vasodilator mechanisms. This is important, as the onset of microvascular disease in diabetics is associated with a marked increase in mortality, to as much as 40-fold in those with clinical nephropathy (urinary albumin excretion $>300 \mathrm{mg} /$ day), compared to those without. ${ }^{33}$

In type I diabetes, no difference in endothelial function and basal NO levels were demonstrated in patients without microvascular disease and non-diabetic controls. ${ }^{33,34}$ Impairment in endothelial function and reduction in basal NO levels, however, were demonstrated in diabetic subjects with microvascular disease (and increased with severity of microvascular disease) compared to diabetics without microvascular disease. The progression of impaired NO associated endothelial dysfunction appears to be a feature of both type 1 and type 2 diabetics with microvascular disease, ${ }^{35}$ but the early increase in arterial compliance and increased basal NO activity may be a specific feature of early INSR.
These findings are in contrast with studies of late type 2 diabetics, women with polycystic ovary disease and obese children where reduced basal arterial compliance has been reported. . $^{35,36}$

In type II diabetic subjects, basal plasma $\mathrm{NO}_{3}^{-}$levels (as a marker of basal nitric oxide levels) were found to be increased compared with nondiabetic controls..$^{29}$ In addition, however, basal plasma $\mathrm{NO}_{3}{ }^{-}$levels appeared to increase with severity of microvascular disease. Caution must be taken in interpreting the latter findings however, as metabolism and renal excretion of $\mathrm{NO}_{3}^{-}$is reduced in advanced microvascular disease (particularly nephropathy), which may be misinterpreted as increased basal NO production. Indeed, no significant difference in $\mathrm{NO}_{2}{ }^{-}$levels (another $\mathrm{NO}$ metabolite measured by the group as a marker of basal nitric oxide levels) were demonstrated between nondiabetic controls, diabetics without microvascular disease, and diabetics with microvascular disease.

In summary, basal NO levels regulate, at least in part, arterial tone. A compensatory increase in basal nitric oxide levels and possibly of non-NO vasodilators such as EDHF ${ }^{32}$ may result in reduced baseline arterial stiffness early in INSR. Eventual depletion of NO may result in increased arterial stiffness, perhaps due to oxidation, which predicts the development of cardiovascular disease during the progression of early INSR to type 2 diabetes.

\section{Angiotensin receptor-mediated responses}

Clinical trials have consistently demonstrated the cardiorenal protective benefits of angiotensin-converting enzyme inhbitors (ACE-I) and AII receptor blockers (ARBs) in high risk cardiovascular patients which are independent of its blood pressure-lowering effect. ${ }^{37-41}$ The precise mechanism for these effects is not certain, although exciting new concepts are emerging. Angiotensin II is the main effector hormone of the renin-angiotensin system. It binds to two main receptor subtypes: the angiotensin II type 1 receptor (AT1R) and the angiotensin II type 2 receptor (AT2R). The AT1R is expressed in various adult tissues, and mediates most of the well-known physiological and pathophysiological effects of AII. AT1R activity in the vasculature is central to the development of increased arterial stiffness which is enhanced in INSR states. ${ }^{7,42}$ AT1R activation in VSMCs have vasoconstrictive, proliferative and inflammatory effects ${ }^{43}$ and is responsible for the accumulation of extracellular matrix proteins such as collagen. ${ }^{44}$ In the endothelium, the AT1R binds and directly alters e-NOS to inhibit its activity. It has potent 
atherogenic effects by up-regulating expression of lectin-like oxidized low-density lipoprotein (LDL) receptor-1 (LOX-1, which induces uptake of oxidized LDL in the endothelial cell), impairing NO production, inducing white blood cell adhesion molecules and stimulating reactive oxygen species (ROS) production, mitogen-activated protein kinase (MAPK) and nuclear factor kappa B (NF-אB) ${ }^{45}$ Recently, the AT1R has been implicated in the inhibition of insulin-signaling pathways in both nondiabetic and diabetic rats, and may thus, itself, facilitate development of insulin resistance. ${ }^{46}$

AT2R expression declines rapidly after birth in humans, but is present in the vascular endothelium and other organs such as the ovary and uterus in adult humans. ${ }^{47}$ Its role in healthy individuals is debated, but there is evidence of up-regulation of AT2R expression during cardiovascular damage, such as in common carotid balloon injury, ${ }^{48}$ hypertension, ${ }^{49}$ myocardial infarction, and heart failure, ${ }^{50,51}$ where it mediates essentially opposing effects to the AT1R. This hypothesis is supported by our studies. In a doubleblinded, placebo-controlled cross-over model, healthy human volunteers were given an intravenous infusion of the highly selective AT2 receptor blocker, PD123319 (10 micrograms/min for 3 minutes). ${ }^{52}$ No significant hemodynamic or arterial stiffness changes were demonstrated after PD123319 infusion, compared with placebo infusion. ${ }^{53}$ In contrast, our study on individuals with HOMA-IR $>2$ (a measure of insulin resistance) and age- and sex-matched NCs given the same dose of intravenous PD123319 (10 micrograms/min for 3 minutes), produced a significantly greater increase in RI, or small to medium sized arterial stiffness with a concurrent rise in systemic vascular resistance index in INSR subjects ${ }^{54}$ while no change occurred in normal controls (NCs). Interestingly, baseline RI levels were lower in the subjects with early INSR consistent with the hypothesis that increased activity of both AT2 and NO medicated mechanisms lead to a reduction in RI in early INSR. This suggests functional expression of AT2R in small to medium sized arteries in patients with early INSR, but not in normal volunteers. The AT2R activity in INSR subjects may be a response to early vascular damage. Evidence that vascular damage occurs in INSR, presumably at a later stage than the subjects that we studied, and causes arterial stiffening was provided by Kim and colleagues who demonstrated reductions in large arterial stiffness indices with improved insulin sensitivity in INSR subjects treated with peroxisome-proliferator-activated receptor $\gamma$ (PPAR- $\gamma)$ agonists. ${ }^{55}$

In diseased vascular beds both AT1R and AT2R expression is upregulated. ${ }^{46}$ Increased vascular sensitivity to AII-induced vasoconstriction has previously been reported in patients with type 2 diabetes mellitus, ${ }^{56}$ suggesting upregulation of AT1R activity in these individuals. In support of this, in a previously described comparative study of subjects with early INSR and age and sex-matched NCs, ${ }^{28}$ infusion of AII $(8,16 \mathrm{ng} / \mathrm{min}$ for 3 minutes) produced a significantly greater increase in RI in INSR subjects. The increase in RI could not be attributed to reduced basal NO levels because, as previously discussed, we have, in fact, demonstrated increased basal NO activity in these subjects. The increase in RI therefore, is most likely attributable to increased AT1R activity in small to mediumsized arteries in subjects with early INSR. In addition, in the same study, we demonstrated evidence of increased AT2R activity in subjects with the INSR syndrome. Infusion of the highly selective AT2R blocker, PD123319 (10, 20 micrograms/min for 3 minutes) produced a significantly greater increase in RI in subjects with early INSR than in NCs where no significant change occurred. Together, these results demonstrate an increased activity of both AT1- and AT2-mediated responses in INSR. An AT2R-mediated increase in basal $\mathrm{NO}^{43,57}$ may, in fact, contribute to the apparent increase in basal NO oxide levels demonstrated after NOS blockade with L-NMMA in these subjects. Further studies with acetylcholine to directly stimulate NO formation may help clarify if there is increased NO formation in the INSR syndrome, independent of the effects of AT2R activation.

In summary, the combined vasodilator effects of increased basal NO levels and increased AT2R expression in INSR subjects are likely to be significant contributors to the observed increased arterial compliance in the early stages of INSR states, and may represent an early response to vascular damage ${ }^{58}$ some of which is induced by increased AT1R expression. AT1R blockade may therefore be a useful treatment for INSR states, with its triple effect of blocking harmful AT1Rmediated vascular effects, increased AT2R expression, ${ }^{59}$ and increased AII formation with unopposed AT2R activation.

\section{Disclosure}

The authors report no conflicts of interest in this work.

\section{References}

1. Cameron AJ, Welborn TA, Zimmet PZ, et al. Overweight and obesity in Australia: the 1999-2000 Australian Diabetes, Obesity and Lifestyle Study (AusDiab). Med J Aust. 2003;178:427-32.

2. Amar J, Ruidavets JB, Chamontin BA, et al. Arterial stiffness and cardiovascular risk factors in a population-based study. J Hypertens. 2001;19:381-7.

3. Benetos A, Rudnichi A, Safar M, et al. Pulse pressure and cardiovascular mortality in normotensive and hypertensive subjects. Hypertension. 1998;32:560-4. 
4. Blacher J, Guerin AP, Pannier B, et al. Impact of aortic stiffness on survival in end-stage renal disease. Circulation. 1999;99:2434-9.

5. Nakanishi N, Suzuki K, Tatara K. Clustered features of the metabolic syndrome and the risk for increased aortic pulse wave velocity in middle-aged Japanese men. Angiology. 2003;54:551-9.

6. Choi KM, Lee KW, Seo JA. Relationship between brachial-ankle pulse wave velocity and cardiovascular risk factors of the metabolic syndrome. Diabet Res Clin Pract. 2004;66:57-61.

7. Zieman SJ, Melenovsky V, Kass D. Mechanisms, pathophysiology, and therapy of arterial stiffness. Arterioscler Thromb Vasc Biol. 2005;25:932-43.

8. Koivistoinen T, Kbi T, Jula A, et al. Pulse wave velocity reference values in healthy adults aged 26-75 years. Clin Physiol Funct Imag. 2007;27:191-6.

9. Lundberg JO. Weitzberg E. Gladwin MT. The nitrate-nitrite-nitric oxide pathway in physiology and therapeutics. Nature Rev Drug Discov. 2008;7:156-67

10. Lloyd-Jones DM, Bloch KD. The vascular biology of nitric oxide and its role in atherogenesis. Аnпи Rev Med. 1996;47:365-75.

11. Vehkavaara S, Groop PH, Seppala-Lindroos A, et al. In vivo endothelial dysfunction characterizes patients with impaired fasting glucose. Diabet Care. 1999;22:2055-60.

12. Avogaro A, Piarulli F, Valerio A, et al. Forearm nitric oxide balance, vascular relaxation, and glucose metabolism in NIDDM patients. Diabetes. 1997;46:1040-6.

13. Goodfellow J, Ramsey M, Luddington LA, et al. Endothelium and inelastic arteries: an early marker of vascular dysfunction in non-insulin dependent diabetes. Br Med J. 1996;312:744-5.

14. Chowienczyk PJ, Kelly RP, MacCallum H, et al. Photoplethysmographic assessment of pulse wave reflection: Blunted response to endothelium-dependent beta2-adrenergic vasodilatation in type 2 diabetes mellitus. J Am Coll Cardiol. 1999;34:2007-14.

15. Darko D, Dornhorst A, Kelly FJ, et al. Lack of effect of oral vitamin $\mathrm{C}$ on blood pressure, oxidative stress and endothelial function in type II diabetes. Clin Sci. 2002;103:339-44.

16. IB Wilkinson, Qasem A, McEniery CM, et al. Nitric oxide regulates local arterial distensibility in vivo. Circulation. 2002;105:213-7.

17. Wilkinson IB, Franklin S, Cockcroft JR. Nitric oxide and the regulation of large artery stiffness: from physiology to pharmacology. Hypertension. 2004;44:112-6.

18. Choi KM, Lee KW, Seo JA. Relationship between brachial-ankle pulse wave velocity and cardiovascular risk factors of the metabolic syndrome. Diabet Res Clin Pract. 2004;66:57-61.

19. Lacy PS, O'Brien DG, Stanley AG, et al. Increased pulse wave velocity is not associated with elevated augmentation index inpatients with diabetes. J Hypertens. 2004;22:1937-44.

20. Kostense PJ, Henry RM, Spijkerman A, et al. Arterial stiffness increases with deteriorating glucose tolerance status: The Hoorn Study Circulation. 2003;16:2089-95.

21. Czernichow S, Bertrais S, Blacher J, et al. Metabolic syndrome in relation to structure and function of large arteries: a predominant effect of blood pressure: a report from the SU.VI.MAX. Vascular Study. Am J Hypertens. 2005;18:1154-60.

22. Salomaa RWV, Kark JD, Nardo C, et al. Arterial disease/ hypertension/ angiotensin system: non-insulin-dependent diabetes mellitus and fasting glucose and insulin concentrations are associated with arterial stiffness indexes: The ARIC Study. Circulation. 1995;91:1432-43.

23. Giannattasio C, Mangoni AA, Failla M, et al. Impaired radial artery compliance in normotensive subjects with familial hypercholesterolaemia. Atherosclerosis. 1996;124:249-60.

24. Hu J, Wallensteen M, Gennser G. Increased stiffness of the aorta in children and adolescents with insulin-dependent diabetes mellitus. Ultrasound Med Biol. 1996;22:537-43.

25. Berry KL, Skyrme-Jones AP, Cameron JD, et al. Systemic arterial compliance is reduced in young patients with IDDM. Am J Physiol. 1999;276:H1839-H45.
26. Mangoni AA, Giannattasio C, Brunani A, et al. Radial artery compliance in young, obese, normotensive subjects. Hypertension. 1995;26:984-8.

27. Laurent S, Hayoz D, Trazzi S, et al. Isobaric compliance of the radial artery is increased in patients with essential hypertension. J Hypertens. 1993;11:89-98

28. Brillante DG, O'Sullivan AJ, Johnstone MT, et al. Arterial stiffness and haemodynamic response to vasoactive medication in subjects with insulin-resistance syndrome. Clin Sci. 2008;114:139-47.

29. Maejima K, Nakano S, Himeno M, et al. Increased basal levels of plasma nitric oxide in type 2 diabetic subjects. Relationship to microvascular complications. J Diabet Complicat. 2001;15:135-43.

30. Woodman RJ, Playford DA, Watts GF. Basal production of nitric oxide (NO) and non-NO vasodilators in the forearm circulation in type 2 diabetes: Associations with blood pressure and HDL cholesterol. Diabet Res Clin Pract. 2006;71:59-67.

31. Graier WF, Wascher TC, Lachner L, et al. Exposure to elevated D-glucose concentrations modulates vascular endothelial cell vasodilatory response. Diabetes. 1993;42:1497-505.

32. Howes LG. Review: The effects of lipid lowering drug therapy on cardiovascular responsiveness in type 2 diabetic patients. Diabet Obes Metabol. 2006;8:8-14.

33. Jensen T, Feldt-Rasmussen B, Bjerre-Knudsen J, et al. Features of endothelial dysfunction in early diabetic nephropathy. Lancet. 1989;333:461-3.

34. Elliott TG, Cockcroft JR, Groop PH, et al. Inhibition of nitric oxide synthesis in forearm vasculature of insulin-dependent diabetic patients: blunted vasoconstriction in patients with microalbuminuria. Clin Sci. 1993;85:687-93

35. Wascher TC, Graier WF, Bahadori B, et al. Time course of endothelial dysfunction in diabetes mellitus. Circulation. 1994;90:1109-10.

36. Cernes R. Zimlichman R. Shargorodsky M. Arterial elasticity in cardiovascular disease: focus on hypertension, metabolic syndrome and diabetes. Adv Cardio. 45:65-81

37. The ONTARGET Investigators. Telmisartan, ramipril, or both in patients at high risk for vascular events. $N$ Eng J Med. 2008;358:1547-59.

38. The HOPE Study Investigators. Effects of an angiotensin-convertingenzyme inhibitor, ramipril, on cardiovascular events in high-risk patients. N Eng J Med. 2000;342:145-53.

39. The SOLVD Investigators. Effect of enalapril on survival in patients with reduced left ventricular ejection fractions and congestive heart failure. N Eng J Med. 1991;325:293-302.

40. Brenner BM, Cooper ME, de Zeeuw D, et al. Effects of losartan on renal and cardiovascular outcomes in patients with type 2 diabetes and nephropathy. N Eng J Med. 2001;345:861-9.

41. Neldam S, Mogensen CE, Tikkanen I, et al. Randomised controlled trial of dual blockade of renin-angiotensin system in patients with hypertension, microalbuminuria, and non-insulin dependent diabetes: the candesartan and lisinopril microalbuminuria (CALM) study. Br Med J. 2000;321:1440-4.

42. Stehouwer CDA, Henry RMA, Ferreira I. Arterial stiffness in diabetes and the metabolic syndrome: a pathway to cardiovascular disease. Diabetologia. 2008;51:527-39.

43. Yan C, Kim D, Aizawa T, et al. Functional interplay between angiotensin II and nitric oxide: cyclic GMP as key mediator. Arterioscler Thromb Vasc Biol. 2003;23:26-36.

44. Ford CM, Li S, Pickering JG. Angiotensin II stimulates collagen synthesis in human vascular smooth muscle cells: involvement of the AT1 receptor, transforming growth factor-beta, and tyrosine phosphorylation. Arterioscler Thromb Vasc Biol. 1999; 19:1843-51.

45. Watanabe T, Barker TA, Berk BC. Angiotensin II and the endothelium: diverse signals and effects. Hypertension. 2005;45:163-9.

46. Igarashi M, Hirata A, Nozaki H, et al. Role of angiotensin II type-1 and type-2 receptors on vascular smooth muscle cell growth and glucose metabolism in diabetic rats. Diabet Res Clin Pract. 2007;75:267-77.

47. Volpe M, Musumeci B, De Paolis P, et al. Angiotensin II AT2 receptor subtype: An uprising frontier in cardiovascular disease? Hypertension. $2003 ; 21: 1429-43$ 
48. Barker TA, Massett MP, Korshunov VA, et al. Angiotensin II type 2 receptor expression after vascular injury: differing effects of angiotensin-converting enzyme inhibition and angiotensin receptor blockade. Hypertension. 2006;48:942-9.

49. Yayama K, Hiyoshi H, Imazu D, et al. Angiotensin II stimulates endothelial NO synthase phosphorylation in thoracic aorta of mice with abdominal aortic banding via type 2 receptor. Hypertension. 2006;48:958-64

50. Blume A, Kaschina E, Unger T. Angiotensin II type 2 receptors: Signalling and pathophysiological role. Curr Opin Nephrol Hyperten. 2001;10:239-46.

51. De Gasparo M, Catt KJ, Inagami $\mathrm{T}$, et al. International union of pharmacology XXIII. The angiotensin II receptors. Pharmacol Rev. 2000;52:415-72.

52. Dean R, Cao Z, Wu L, et al. Role of angiotensin receptor subtypes in mesenteric vascular proliferation and hypertrophy. Hypertension. 1999; 34:408-14.

53. Brillante DG, Johnstone MT, Howes LG. Effects of intravenous PD123319 on haemodynamic and arterial stiffness indices in healthy volunteers. J Renin Angiotens Aldo Syst. 2005;6:102-6.
54. Brillante DG, O'Sullivan AJ, Johnstone MT, et al. Evidence for functional expression of vascular AT2 receptors in patients with insulin resistance. Diabet Obes Metab. 2008;10:143-50.

55. Kim SG, Ryu OH, Kim HY, et al. Effect of rosiglitazone on plasma adiponectin levels and arterial stiffness in subjects with pre-diabetes or non-diabetic metabolic syndrome. Eur J Endocrinol. 2006; 154:433-40.

56. Weidmann P, Beretta-Piccoli C, Trost BN. Pressor factors and responsiveness in hypertension accompanying diabetes mellitus. Hypertension. 1985;7(6 Pt 2):I133-42.

57. Carey RM, Jin XH, Wang ZQ, et al. Nitric oxide: A physiological mediator of the type 2 (AT2) angiotensin receptor. Acta Physiol Scand. 2000;168:65-71.

58. Woodman RJ. Does compensatory nitric oxide and angiotensin II receptor activity reduce arterial stiffness in early-stage insulin resistance? Clin Sci. 2008;114:119-21.

59. Savioa C, Touyz RM, Volpe M, et al. Angiotensin Type 2 Receptor in resistance arteries of type 2 diabetic hypertensive patients. Hypertension. 2007;49:341-6. 